\title{
Programas de transferencias monetarias condicionadas: experiencias en América Latina
}

\author{
Pablo Villatoro
}

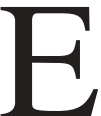

n este artículo se analiza la experiencia de cinco programas de transferencias monetarias condicionadas en América Latina que apuntan a reducir la pobreza y fomentar el capital humano en educación, salud y nutrición. Estos programas innovadores pueden contribuir al logro de los Objetivos de Desarrollo del Milenio, y algunos han llegado a ser componentes fundamentales de las políticas antipobreza de sus respectivos países. En este artículo se presentan en primer lugar algunos conceptos que fundamentan la implementación de los programas de transferencias condicionadas. A continuación, se examinan las experiencias siguientes: Programa Bolsa Escola y Programa para la Erradicación del Trabajo Infantil (Brasil); Familias en Acción (Colombia); Red de Protección Social (Nicaragua), y Programa Oportunidades (México), analizando sus objetivos, componentes, mecanismos de focalización, impactos y eficiencia. Finalmente, se plantean recomendaciones de política y se identifican elementos de diseño que podrían constituirse en buenas prácticas al momento de aplicar este tipo de programas. 


\section{Introducción}

En la Cumbre del Milenio se establecieron como metas de desarrollo para el 2015, entre otras, la reducción a la mitad de la pobreza y el hambre, la universalización de la educación primaria, el acceso equitativo a todos los niveles de educación y la reducción de la mortalidad materno-infantil. Para cumplir con estas metas se requiere el compromiso del sector público en la implementación de estrategias multisectoriales que contribuyan simultáneamente al logro de los objetivos propuestos (Naciones Unidas, 2001).

Este artículo analiza las experiencias de cinco programas de transferencias monetarias condicionadas en América Latina que apuntan a reducir la pobreza y fomentar el capital humano en educación, salud y nutrición, y que pueden constituirse en instrumentos relevantes para el logro de las metas de desarrollo de las Naciones Unidas. Estas experiencias fueron selecciona- das por razones de disponibilidad de bibliografía y no necesariamente porque representen las mejores prácticas en este tipo de programas.

Después de esta sección introductoria, en la sección II se presentan los conceptos que sustentan la implementación de los programas de transferencias condicionadas. En la sección III se analizan las experiencias del Programa Bolsa Escola (de becas escolares) y el Programa de Erradicación del Trabajo Infantil, ambos de Brasil; el programa Familias en Acción, de Colombia; la Red de Protección Social, de Nicaragua, y el Programa Oportunidades, de México, en lo que se refiere a sus objetivos, componentes, mecanismos de focalización, impactos y costo-efectividad. Finalmente, en la sección IV se formulan algunas recomendaciones de política y se identifican elementos de diseño que podrían ser útiles al momento de implementar este tipo de programas.

\section{II}

\section{Conceptos preliminares}

Los programas que condicionan las transferencias monetarias a la inversión en capital humano deben ser entendidos en el contexto de un nuevo enfoque de la protección social. En rigor, las consecuencias sociales de las crisis económicas y de los programas de ajuste estructural han generado un debate en torno a la racionalidad de esa protección (Ravallion, 2003). Del debate se desprende que el sistema de protección ha evolucionado desde una perspectiva centrada en la reducción de la pobreza en el corto plazo, hacia un enfoque de manejo de riesgos, que tiene como objetivo acrecentar el capital humano y superar la pobreza en el largo plazo.

Según el enfoque de manejo de riesgos sociales (Holzmann y Jorgensen, 2000), las personas, hogares y comunidades están expuestos a múltiples riesgos. La pobreza implica una mayor vulnerabilidad, ya que los pobres tienen poco acceso a instrumentos adecuados para manejar riesgos y se encuentran en peores condiciones para afrontar situaciones de crisis. Los mecanismos más utilizados por las familias pobres para enfrentar las perturbaciones económicas son las estrategias informales (por ejemplo, sacar a los niños de la escuela), cuya ineficiencia puede reducir de manera irreversible el capital humano y perpetuar el ciclo intergeneracional de la pobreza.

El modelo de manejo de riesgos asigna tres funciones a la política pública: prevención, mitigación y enfrentamiento. El sistema de protección social desarrolla estrategias de mitigación, orientadas a reducir el impacto de un riesgo futuro, y de enfrentamiento, que buscan atenuar el impacto del riesgo una vez que este se ha generado (Banco Mundial, 2001c; Hicks y Wodon, 2001). Desde esta lógica, las acciones de protección social constituyen inversiones en capital humano que fomentan el acceso a servicios básicos y evitan estrategias con consecuencias adversas en el largo plazo, y que benefician a: i) las personas que viven en pobreza estructural, ii) a las que se encuentran levemente por sobre la línea de pobreza, y iii) a los grupos con necesidades especiales. 
Los programas de transferencias condicionadas se enmarcan en el concepto de protección social como inversión en capital humano. Tienen como premisa que la reproducción intergeneracional de la pobreza se debe a la falta de inversión en capital humano, y buscan, mediante el condicionamiento de las transferencias, generar incentivos para esa inversión (CEPAL, 2000). Las transferencias condicionadas podrían ser más eficaces que las intervenciones tradicionales porque el condicionamiento reduciría el costo de oportunidad de la escolarización; esto a su vez reforzaría el efectoingreso de la transferencia, dado que la asistencia a la escuela y el trabajo infantil son sustitutos entre sí (Skoufias y Parker, 2001).

Skoufias y Parker (2001) proponen un modelo económico para comprender en qué forma las transferencias condicionadas interactúan con las preferencias y restricciones de ingresos de las familias. Este modelo señala que las condiciones que plantea el programa afectarán de diversa manera la asignación de tiempos en la familia, lo que dependerá de las preferencias del hogar y de su posición presupuestaria anterior a la implementación del programa. En los hogares para los cuales las condiciones del programa sean vinculantes (los niños deben ir a la escuela), el programa tendría efectos de agregación y sustitución del ingreso que reforzarían su impacto. Entre los hogares que no estén en esa situación, la intervención sólo tendría efectos en los ingresos monetarios.

Si un niño destina más tiempo al trabajo y menos a la escuela, la familia podrá consumir bienes adicionales, cuyo valor monetario será igual al salario de mercado que se paga por el trabajo infantil. En cambio, si un niño deja de usar su tiempo en actividades laborales y asiste a la escuela, serán menos los bienes adicionales que podrá consumir el grupo familiar. Si se relacionan estas asignaciones presupuestarias con el supuesto de maximización de la utilidad, se puede concluir que habrá un monto mínimo de transferencia que resultará en incentivos $>0$ para enviar los niños a la escuela. Para Skoufias y Parker (2001), las transferencias condicionadas de montos superiores a este mínimo tendrían una alta probabilidad de lograr efectos positivos en la escolarización de los niños.

Entre las principales limitaciones que podrían tener las transferencias condicionadas, se ha señalado la no consideración del eslabón empleo en la cadena de reproducción intergeneracional de la pobreza. La CEPAL (2002) y Britto (2005) han advertido que no se puede garantizar la conversión de mayores logros educativos en un incremento de los ingresos monetarios en el largo plazo, debido a que esta relación está mediada por variables como la calidad de la oferta educativa, la rentabilidad de la educación y el desempleo.

También se ha planteado que estos modelos no incorporan los factores culturales y psicosociales que podrían incidir en las elecciones y preferencias de los sujetos, y que no toman en cuenta las imperfecciones de los mercados. Como indica la CEPAL (2000), constituyen obstáculos a las intervenciones de subsidio a la demanda: i) que los padres de familia no siempre actúan como consumidores racionales; ii) que la oferta en el mercado educacional no es transparente, y iii) que la educación no puede ser considerada exclusivamente como un bien de consumo.

Desde una perspectiva más empírica, una mayor asistencia del niño a la escuela no siempre implicaría una reducción en la incidencia e intensidad de todas las clases de trabajo infantil. Por ejemplo, al evaluar el impacto del programa de transferencias condicionadas de Bangladesh se encontró que el aumento de la asistencia escolar tuvo lugar a expensas de la disminución del tiempo libre de los niños (Ravallion y Wodon, 1999). También es importante saber si los programas provocan cambios en las actitudes de las familias ante la educación y el trabajo infantil, ya que si no hay modificaciones en este ámbito los efectos de las intervenciones podrían no ser sostenibles en el largo plazo.

Al mismo tiempo, el incremento de la demanda de educación podría generar externalidades negativas si la oferta se mantuviera constante, lo que indica la necesidad de establecer subsidios compensatorios a la oferta. Algunos autores han señalado asimismo la importancia de analizar cómo reciben los actores institucionales del sistema escolar la nueva demanda generada por las transferencias, y de establecer si los subsidios a la demanda contribuyen a mejorar el aprendizaje de los niños más pobres, y también se han preguntado si la expansión de la oferta educacional podría ser una vía más costo-efectiva que las transferencias condicionadas para mejorar el acceso a la educación e incrementar el capital humano de las familias pobres (Coady y Parker, 2002; Skoufias y Parker, 2001).

En cuanto a los ingresos monetarios, se ha sostenido que los efectos de las transferencias condicionadas podrían ser limitados, debido a la situación de extrema vulnerabilidad de las familias que reciben los beneficios (por ejemplo, las intervenciones podrían ser eficaces para disminuir la brecha de pobreza, pero no para superarla), y que las transferencias monetarias no siempre ayudarían a mejorar la calidad de la alimentación de los más pobres. La literatura 
muestra elasticidades calorías-ingreso que oscilan entre valores bastante altos, de 0,54, y muy bajos, de 0,01 (Hoddinot y Skoufias, 2003); esto sugiere que se debe analizar la varianza adicional a las transferencias que podrían aportar intervenciones de carácter psicosocial y cultural encaminadas a fomentar actitudes favorables a un consumo alimentario de mejor calidad.

También resulta de interés analizar los datos sobre la eficiencia y eficacia de las diferentes modalidades de implementación de los programas de transferencias condicionadas, en lo que se refiere a la incorporación de distintos componentes (intervenciones unidimensionales o multidimensionales), al tipo de gestión (centralizada o descentralizada) y a los mecanismos de focalización. Es igualmente importante conocer la participación de la sociedad civil en los espacios de toma de decisiones y de prestación de servicios, y estudiar de qué manera se aborda la atención a poblaciones más complejas, como los niños trabajadores de la calle.

El análisis de las cinco experiencias de transferencias monetarias condicionadas que se hace en la sección siguiente considera los elementos conceptuales planteados en los párrafos anteriores, recoge y sistematiza la evidencia disponible, e intenta entregar herramientas para el debate como aporte a las prácticas de implementación de este tipo de programas.

\section{III}

\section{Experiencias de transferencias monetarias} condicionadas en América Latina

\section{El Programa Nacional de Bolsa Escola (Brasil)}

Este programa de becas escolares fue implementado inicialmente por el gobierno estadual de Campinhas en 1995. Ese mismo año se comenzó a aplicar una nueva versión en el Distrito Federal de Brasilia. En los años siguientes, el programa se expandió con rapidez: en el 2001 eran siete los estados y más de 200 los municipios que llevaban a cabo intervenciones tipo Bolsa Escola (Godoy, 2004). Posteriormente, estos programas pasaron a depender del Ministerio de Educación, y en el 2003, en el contexto de la nueva estrategia nacional antipobreza Fome Zero, fueron incluidos en el programa federal Bolsa Familia, que centralizó la administración de todos los dispositivos de transferencias condicionadas.

El Programa Bolsa Escola fue creado para extender la permanencia en la educación primaria y secundaria de niños de 6 a 15 años de edad, matriculados en algún establecimiento de educación primaria o secundaria, y pertenecientes a familias con ingresos mensuales per cápita de hasta 90 reales; mediante las transferencias, el programa fomenta la asistencia a la escuela y procura disminuir el trabajo infantil (Secretaria do Programa Nacional de Bolsa Escola, 2002). Se espera que el incremento del capital humano educacional de los niños permita reducir la pobreza en el largo plazo (Banco Mundial, 2001a).
Dicho programa tiene un componente, de educación, mediante el cual se hacen transferencias monetarias a familias pobres con niños en edad escolar. Las transferencias se entregan con la condición de que los niños asistan a la escuela como mínimo el $85 \%$ de la jornada escolar en el mes (Secretaria do Programa Nacional de Bolsa Escola, 2002). Los montos de las transferencias no dependen del número, la edad o el género de los niños de las familias beneficiarias, y varían mucho de un municipio a otro (Banco Mundial, 2001a).

Se ha sugerido que un ajuste de los montos de las transferencias conforme a las características de las familias atendidas podría mejorar la eficiencia y eficacia del programa (Banco Mundial, 2001a). Por ejemplo, el costo de oportunidad de la asistencia a la escuela de los niños adolescentes podría ser superior al de los niños de edades más bajas, lo que reduciría el incentivo para enviar a los adolescentes a la escuela (Skoufias y Parker, 2001). Según el Banco Mundial (2001a), el monto de la transferencia debería determinarse atendiendo a la jerarquización de los objetivos del programa (paliación o superación de la pobreza).

Los procesos de selección de beneficiarios del Programa Bolsa Escola se hallaban inicialmente en manos de las municipalidades. Dentro de estas, el programa elegía primero a las localidades más pobres y luego seleccionaba a sus familias más vulnerables, mediante un sistema de puntaje que integraba distintos 
indicadores de los estándares de vida familiar. El programa no disponía en sus comienzos de un sistema de focalización nacional, que permitiera optimizar la selección de las áreas geográficas más pobres y eliminar ineficiencias administrativas (Banco Mundial, 2001a).

Posteriormente, el gobierno brasileño estableció un catastro nacional de familias beneficiarias de programas federales, con el propósito de mejorar la focalización. En el 2002, el proceso de selección de beneficiarios abarcaba el levantamiento de datos por los municipios, la selección de familias elegibles por una instancia del gobierno federal, y la elección definitiva de las familias beneficiarias desde el ámbito local con la participación de Consejos de Control Social, que incluyen a representantes de organizaciones de la sociedad civil (Secretaria do Programa Nacional de Bolsa Escola, 2002).

La evidencia sugiere que el programa ha sido exitoso en la focalización, debido a que las tasas de fuga han sido más bien bajas. Sin embargo, los indicadores de subcobertura (la población objetivo que no es alcanzada por el programa) han sido altos, lo que se atribuye a los escasos recursos financieros de las municipalidades más pobres (Banco Mundial, 2001a). Además, se detecta la exclusión de las familias con niños preescolares y adolescentes. Se han sugerido dos alternativas para la incorporación de estos grupos: la implementación de programas paralelos, o bien la integración de estos segmentos al Programa Bolsa Escola, lo que implicaría un componente nutricional y de salud adicional (Banco Mundial, 2001a).

Uno de los principales problemas para la incorporación de las poblaciones no cubiertas es el financiamiento: como los programas son financiados principalmente con recursos locales, los municipios más pobres no pueden implantarlos. Específicamente, se ha sugerido la entrega de recursos adicionales a los municipios que cuentan con menos recursos, dado que los recortes en los montos de las transferencias podrían comprometer la eficacia del Programa Bolsa Escola (Banco Mundial, 2001a). Sin embargo, en los últimos años se ha tendido a incrementar la cobertura mediante la reducción de los montos transferidos (Godoy, 2004).

También se han planteado objeciones en torno a los límites de permanencia definidos por dicho programa (entre 1 y 2 años), los que reflejarían una falta de garantías en términos de la continuidad del beneficio. $\mathrm{La}$ imposición de estos umbrales de permanencia se atribuye a que la mayor parte de la legislación de programas de beca escolar no tiene como propósito "que los niños concluyan la enseñanza fundamental, sino sacarlos de las calles, lo que refuerza un carácter asistencialista del programa en detrimento de su impacto redistributivo" (Godoy, 2004, p. 22).

Con relación a los impactos del Programa Bolsa Escola en la acumulación de capital humano, la evidencia muestra resultados positivos en la asistencia escolar y en la reducción de la brecha edad/año escolar. Algunos estudios han mostrado que la asistencia a la escuela es más alta y la deserción es más baja en los beneficiarios del programa que en grupos no atendidos, y que más niños beneficiarios ingresan a la escuela a la edad adecuada con relación a los sujetos de grupos de comparación (Banco Mundial, 2001a). Se ha señalado que el Programa Bolsa Escola ha contribuido a desmontar los mecanismos usados por las escuelas para excluir a los estudiantes más pobres, debido a que en circunstancias normales, las escuelas - con el pretexto de criterios académicos - tienden a excluir a los estudiantes más vulnerables (Banco Mundial, 2001a).

Una pregunta que ha recibido poca atención es si los programas de transferencias condicionadas a la inversión en capital humano educativo tienen incidencia en los resultados de aprendizaje. Igualmente es importante disponer de información sobre los procesos de recepción en las escuelas de la demanda adicional creada por el programa. Para responder a estas preguntas, se efectuó un estudio cuasi experimental con dos mediciones (antes del ingreso al programa y un año después) sobre una muestra de niños de familias beneficiarias de Bolsa Escola y un grupo de comparación, integrado por niños de familias no incluidas en el programa (Banco Mundial, 2001a).

La investigación mostró que las percepciones de los profesores y directores de las escuelas sobre el programa eran favorables. Estos actores consideraron que la intervención del Programa Bolsa Escola reforzaba su acción educativa, y que proporcionaba oportunidades para mejorar su trabajo con los niños más pobres y elevar sus niveles de aprendizaje. Sin embargo, el estudio no mostró diferencias en los niveles de aprendizaje entre los sujetos del programa y los del grupo de comparación (Banco Mundial, 2001a). Estos datos indican que es necesario desarrollar acciones adicionales para mejorar la calidad de la oferta.

$\mathrm{Al}$ mismo tiempo, no existe evidencia concluyente que permita afirmar que el Programa Bolsa Escola reduce el trabajo infantil (Banco Mundial, 2001a). En todo caso, si se supone que el programa ha incrementado la asistencia a la escuela, se puede sostener que ha disminuido las horas disponibles para el trabajo de 
los niños. En este campo existe un doble desafío: evitar el trabajo infantil en las horas no cubiertas por la escuela, y analizar la capacidad de diferentes montos de transferencias condicionadas para incentivar la reducción del trabajo infantil.

Por otra parte, en estudios seccionales se ha observado que el programa aminora la brecha de pobreza que afecta a las familias beneficiarias (Banco Mundial, 2001a), pero la evidencia sobre la reducción de la pobreza no es consistente. Por ejemplo, según el Banco Mundial (2001a), el Programa Bolsa Escola disminuye la prevalencia de la pobreza. Sin embargo, para Godoy (2004), las becas escolares han tenido poco impacto en la reducción de la pobreza, lo que se explica por el bajo monto de las transferencias y la precaria situación de las familias beneficiarias.

Godoy (2004) también señala que el Programa Bolsa Escola no ha contribuido a mejorar las capacidades de generación de ingresos autónomos de las familias. En una encuesta a familias beneficiarias de Campinhas, se observó que apenas el $1 \%$ de los sujetos que se desvincularon del programa lo hicieron por haber obtenido autonomía económica, y que entre todas las familias que dejaron el programa, ninguna resolvió sus problemas económicos. Según Godoy, esta situación es atribuible a la escasa articulación de las intervenciones del Programa Bolsa Escola con las iniciativas de capacitación e inserción laboral.

\section{El Programa para la Erradicación del Trabajo Infantil (PETI), de Brasil}

Este programa fue creado por el gobierno de Brasil con el propósito de erradicar las peores formas de trabajo infantil en las zonas rurales. A inicios de 1999, el PETI comenzó a expandirse a áreas urbanas para enfrentar el trabajo de los niños de la calle, teniendo como población objetivo 266 mil niños de 10 a 14 años de edad que desarrollan actividades como la prostitución, el tráfico de drogas, la recolección de basura y la venta callejera. En los últimos años, el gobierno brasileño unificó el PETI con el Fondo de Garantía de la Renta Mínima, creando el programa Alborada, para mejorar la complementariedad, eficiencia y eficacia de las intervenciones.

El PETI tiene objetivos similares a los del Programa Bolsa Escola, ya que apunta a elevar el logro educacional y acrecentar la reducción de la pobreza, pero su principal propósito es erradicar el trabajo infantil. Para lograr esta meta, hace transferencias a familias con niños en edad escolar (7 a 14 años) de zonas rura- les y urbanas, con la condición de que ellas garanticen que los niños asistirán a un $80 \%$ de las horas de escuela y que participarán en el subprograma Jornada Ampliada, en el que se desarrollan actividades después de la escuela. Los montos de las transferencias son más altos en las zonas urbanas que en las rurales, difieren de un estado a otro y en algunas ocasiones dependen del número de niños por familia. Las transferencias son entregadas mayoritariamente a la madre.

El programa también tiene un componente psicosocial, orientado a promover cambios en las creencias y actitudes prevalecientes en muchas familias pobres, cuya precaria situación lleva a que perciban como bajos los beneficios de la educación y tengan una alta valoración del trabajo infantil. Al mismo tiempo, el PETI entrega recursos a los municipios para el financiamiento del subprograma Jornada Ampliada, con el fin de evitar que los niños asistan a la escuela y a la vez trabajen. Por lo tanto, este subsidio a la oferta busca limitar el tiempo disponible para el trabajo infantil.

En el proceso de focalización que emplea el PETI, primero se seleccionan las zonas que evidencian las incidencias más altas de trabajo infantil, y luego, dentro de las localidades, se recolecta información sobre los hogares. Estos datos son remitidos al nivel federal, ámbito en el que se realiza una preselección de familias elegibles. Finalmente, la población beneficiaria es seleccionada en el nivel local, con la participación de representantes de la sociedad civil. Este procedimiento tiene dos ventajas: i) minimiza el error de exclusión y ii) permite implementar el programa en zonas que no cuentan con medios suficientes.

Existe alguna evidencia que indica que el programa en zonas rurales está correctamente focalizado. Por ejemplo, se ha observado que las familias beneficiarias tienden a ser bastante grandes (hasta siete integrantes), y que un tercio de las madres nunca asistió a la escuela. En un estudio cualitativo realizado en Pernambuco, la mayor parte de los beneficiarios afirmó que gastaba las transferencias en bienes de primera necesidad, como ropa y alimentos (Banco Mundial, 2001b).

El PETI comenzó en 1999 a trabajar para la erradicación de las peores modalidades de trabajo infantil en zonas urbanas. Con esto han aparecido dificultades vinculadas al diseño del programa, sus mecanismos de focalización, la determinación de los montos de sus transferencias y la selección de sus componentes. Primero, los datos sobre los niños trabajadores de la calle en zonas urbanas son poco confiables o inexistentes, lo que hace más compleja la focalización. Segundo, por la heterogeneidad del trabajo infantil 
urbano la especificación del monto de las transferencias es más compleja que en las zonas rurales. Tercero, los problemas del trabajo infantil callejero están vinculados al abandono familiar, la violencia, las drogas y las vulnerabilidades de salud, por lo cual es indispensable incorporar en el programa componentes psicocomunitarios y de salud.

Hasta ahora, las intervenciones psicosociales del PETI ante la prostitución infantil han sido preventivas y de asistencia, y se han focalizado en niños y niñas de 7 a 14 años provenientes de familias con ingresos per cápita menores a la mitad del salario mínimo. Entre las acciones preventivas, cabe mencionar una campaña nacional para generar conciencia social sobre el problema y la realización de seminarios y talleres. Las medidas tendientes a mejorar la asistencia a la escuela incluyeron intervenciones para facilitar el acceso de los niños y niñas a las redes institucionales de apoyo y para fomentar la interrelación entre las familias, las escuelas y la comunidad. El PETI no ha llevado a cabo acciones destinadas a reducir el trabajo doméstico infantil en zonas urbanas.

Se ha propuesto que el PETI incorpore experiencias de organizaciones no gubernamentales (ONG), porque el modelo PETI es consistente con las metodologías utilizadas por esas organizaciones (Banco Mundial, 2001b). En particular, se ha sugerido la conveniencia de que tome medidas encaminadas a mejorar la autoestima y las habilidades sociales de los niños, y que implemente acciones educativas en salud y derechos humanos. Una alternativa a la incorporación de nuevos componentes al PETI podría ser la de condicionar las transferencias a la participación de los niños en programas preventivos y de asistencia llevados a cabo por ONG. De esta manera se evitarían los costos de agregar un nuevo componente, y se utilizaría la experiencia de estas organizaciones.

Con relación a los efectos del PETI, solamente se dispone de datos sobre el programa rural. En rigor, se efectuó una evaluación del impacto del PETI mediante una encuesta que incluyó a tres municipalidades participantes en el programa y tres no participantes (Banco Mundial, 2001b). Esta encuesta recopiló información sobre los efectos del programa en el número de horas escolares, los años de educación alcanzados, la relación edad/años de escolaridad, el trabajo infantil y la participación de los niños en actividades laborales de riesgo.

Los resultados de la evaluación muestran que el PETI duplicó las horas dedicadas a la escuela y redujo significativamente el trabajo infantil. Según Sedlacek,
Yap y Orazem (2002), lo que explicó la menor participación de los niños en la fuerza de trabajo fue el programa Jornada Ampliada. Sin embargo, estos autores señalan que el PETI tuvo menos éxito en disminuir el número de horas de trabajo infantil. En los estados de Bahía y Sergipe se apreció un "efecto derrame" que se tradujo en un incremento de la probabilidad de trabajo infantil en niños no incluidos en el programa.

También se han realizado estudios para establecer los efectos del componente psicosocial del PETI en las actitudes de los adultos hacia la educación y el trabajo infantil de los niños. En general, una minoría de los adultos beneficiarios opina que es necesario que los niños vayan a la escuela (18\% en Bahía y $11 \%$ en Pernambuco), y más de la mitad no cree que el trabajo infantil sea negativo para el desarrollo de los niños. Estas cifras se ven corroboradas con lo sucedido en algunos municipios que se han atrasado en la entrega de las transferencias: ante este evento, algunos beneficiarios amenazan con sacar a sus niños de la escuela, lo que revela la dificultad de cambiar las creencias sobre la educación y el trabajo infantil (Banco Mundial, 2001b).

En cuanto a los efectos en la situación de la mujer, algunos estudios han mostrado que el hecho de que el programa entregue la transferencia a la madre permite que las mujeres adquieran mayor responsabilidad, independencia y protagonismo en la familia. Las mujeres encuestadas señalaron mayoritariamente que sus vidas cambiaron después de comenzar a recibir las transferencias, porque pudieron destinar más tiempo al trabajo como resultado de la asistencia a la escuela de sus hijos (Banco Mundial, 2001b).

\section{El programa Familias en Acción, de Colombia}

Este programa fue aplicado en el marco de la creación en 1999 de la Red de Apoyo Social (RAS), una red temporal de seguridad social desarrollada por el gobierno colombiano para paliar las consecuencias de la recesión económica y de los ajustes de política fiscal en el bienestar de las poblaciones más vulnerables. Junto a Familias en Acción, fueron puestos en marcha otros dos programas sociales: Jóvenes en Acción y Empleo en Acción.

Familias en Acción es un dispositivo de transferencias monetarias condicionadas, destinado inicialmente a aminorar el impacto de la recesión en los hogares pobres. El objetivo específico del programa es proteger y promover la formación de capital humano en niños de 0 a 17 años de edad pertenecientes 
a hogares en situación de pobreza, mediante el apoyo y fomento de las inversiones de las familias pobres en salud, nutrición y educación.

Al igual que el Programa Bolsa Escola y el PETI, Familias en Acción presta asistencia mediante un componente de educación, pero a este agrega servicios de salud y nutrición. El componente educación entrega una transferencia que equivale al costo directo que supone para las familias más pobres el hecho de mandar a sus niños de 7 a 18 años a la escuela. Este monto alcanzaba a 6 dólares mensuales per cápita en los establecimientos de educación primaria, y a 12 dólares en las escuelas secundarias (Rawlings, 2002). El monto de la transferencia no está determinado por el costo de oportunidad del trabajo infantil, lo que podría deberse tanto a que el programa no tiene metas explícitas en ese ámbito como a los objetivos de enfrentamiento de la crisis.

El segundo componente — salud y nutricióntransfiere recursos a familias pobres con niños de 0 a 6 años de edad. Las ayudas monetarias se entregan a la madre de familia, para garantizar que sea invertida en el niño, y también para dar más autonomía a las mujeres.

En cuanto a las condiciones que impone el programa, en el componente de salud y nutrición la familia mantendrá los beneficios siempre y cuando lleve a los niños a los servicios de salud primaria para los controles del crecimiento y las inmunizaciones correspondientes. En el componente educación, las transferencias se suspenderán si los niños presentan una tasa de inasistencias injustificadas superior al 20\% y/o si repiten más de un año escolar.

Desde el punto de vista de los mecanismos de focalización, Familias en Acción atiende zonas rurales y urbanas no cubiertas por el programa Empleo en Acción. Estas áreas deben cumplir con criterios básicos de elegibilidad, como la presencia de un banco y la existencia de una oferta adecuada en materia de educación y salud. Dentro de las localidades que cumplen con las condiciones de elegibilidad, el programa selecciona a familias clasificadas como SISBEN 1 , que corresponden a los grupos más vulnerables. No se da apoyo en salud y nutrición a las familias SISBEN 1 que tengan a sus hijos de 0 a 6 años de edad en Hogares Comunitarios de Bienestar.

Familias en Acción ha mostrado un buen desempeño en lo que se refiere a minimizar los errores de inclusión. Un $71 \%$ de las familias que ingresó al programa estaba en situación de indigencia al momento de iniciarse la intervención (Attanasio, Meghir y otros,
2004). Sin embargo, ha habido problemas para cubrir áreas rurales aisladas que carecen de servicios sociales y financieros, lo que configura una probabilidad importante de error de exclusión.

El programa cuenta con un sistema de evaluación de impacto que comenzó a aplicarse en el 2002. En ese año se empezó a recoger información sobre tres grupos de población: i) familias con tres meses de intervención; ii) familias por intervenir y, iii) familias para las cuales no hay una intervención planificada. El diseño de la evaluación considera la realización de mediciones repetidas de seguimiento a los tres grupos seleccionados (Departamento Nacional de Planeación, 2003).

Los primeros informes de evaluación muestran efectos favorables del programa en las áreas de educación, salud y nutrición. Tuvo impactos positivos en la asistencia a la escuela entre los niños rurales y urbanos de 12 a 17 años, pero no entre los de 8 a 11 años. Según Attanasio, Battistin y otros (2005), esta situación se explica porque los niños de menor edad tenían tasas de asistencia a la escuela muy altas antes de ingresar al programa.

En salud, Familias en Acción incrementó significativamente el porcentaje de niños menores de 48 meses con controles preventivos al día y redujo la proporción de niños rurales con diarrea. En la dimensión nutricional, el programa aumentó considerablemente el consumo de alimentos, principalmente en zonas rurales. La mayor parte del incremento se debió al consumo de una mayor variedad de alimentos, especialmente de aquellos ricos en proteínas (Attanasio, Battistin y otros, 2005).

\section{La Red de Protección Social (RPS), de Nicaragua}

Este programa promueve el desarrollo del capital humano educacional, nutricional y de salud en familias rurales extremadamente pobres. El diseño de la RPS contempla una fase piloto, que considera dos etapas. En la primera etapa, el programa ha beneficiado a 6.000 familias de 21 áreas censales, seleccionadas mediante criterios de focalización geográfica y de hogares. En la segunda etapa, la RPS beneficiará a 4.000 familias.

El programa tiene dos componentes: i) salud/seguridad alimentaria y ii) educación. Interviene mediante transferencias monetarias y otros servicios destinados a incentivar la demanda y a mejorar la oferta en educación y en salud. En el componente salud/seguridad alimentaria, la transferencia se hace para garantizar la compra de alimentos por familias pobres con 
niños menores de 5 años, beneficio que está condicionado a la asistencia de la madre a talleres de salud y a la concurrencia de los niños a controles médicos.

Los talleres para las madres se abocan a capacitarlas en habilidades básicas en materia de nutrición y salud reproductiva. Por medio de controles médicos, se entregan servicios de vigilancia del crecimiento y de vacunación y se proporcionan vitaminas y antiparasitarios a los niños. También se realizan visitas preventivas mensuales a los niños menores de 2 años. Al mismo tiempo, se capacita a prestadores de atención en salud de organizaciones no gubernamentales, para asegurar la entrega de servicios médicos de calidad a las comunidades beneficiarias.

En el componente educación, el programa entrega a familias pobres con niños de 7 a 13 años de edad una transferencia monetaria condicionada a que los niños se matriculen y cumplan con un $85 \%$ de la asistencia a la escuela. Este beneficio consiste en un monto fijo por hogar, que no varía según el número de niños en la familia. A esto se agrega una transferencia para la compra de bienes necesarios para la escuela (ropa, útiles escolares), cuyo monto sí se determina conforme al número de niños en la familia.

El componente educación también considera un subsidio a la oferta, el que se expresa en una pequeña transferencia a los profesores. En ocasiones, las organizaciones de apoderados solicitan apoyo monetario para afianzar el trabajo de los profesores. También es frecuente que se entregue esta ayuda económica a las familias, las cuales hacen llegar los montos correspondientes a los profesores. Estos últimos pueden emplear los recursos para la compra de artículos escolares y guardar una parte de la transferencia para uso posterior.

La transferencia para alimentos alcanza a un 13\% del gasto total anual de las familias beneficiarias antes de su ingreso al programa, y el apoyo monetario para la educación representa un $8 \%$ del gasto anual familiar. La transferencia total equivale nominalmente a un incremento de $21 \%$ del consumo de las familias, cifra comparable a la asistencia proporcionada por el Programa Oportunidades en México y el Programa de Asignación Familiar (PRAF) en Honduras. Pero a diferencia de esos dos programas, el aporte de la RPS no se ajusta según las tasas de inflación, de manera que ha experimentado caídas de aproximadamente $7 \%$ en el valor real de las transferencias (IFPRI, 2002).

Para poner en marcha la RPS hubo esfuerzos de coordinación en los niveles nacional, regional y local. En el ámbito local, la planificación fue efectuada por comités en los que participaron funcionarios ministe- riales, representantes de la sociedad civil y personal del RPS. Esta colaboración resultó importante para adecuar la oferta, en un escenario de incremento de la demanda de atención en salud y educación.

En la fase piloto de la RPS, las transferencias se han entregado generalmente a la madre de familia, debido a que la administración de los recursos por las mujeres lleva a mejores resultados en la seguridad alimentaria de la familia y en la calidad de vida de los niños (IFPRI, 2002). En ocasiones se hizo una transferencia adicional a las familias que cumplieron con todos los compromisos y metas educacionales y de salud.

En la fase piloto de la RPS se realizó una evaluación de impacto que contempló un diseño cuasi experimental, con grupos de intervención y control, mediciones de la línea de base y seguimiento. El estudio permitió estimar los principales efecto del programa en los ingresos, la alimentación, la nutrición y la educación (IFPRI, 2002).

La RPS tuvo un impacto de $20 \%$ en el gasto per cápita de los hogares beneficiarios, lo que se explica principalmente por una contracción de los ingresos en el grupo de control. En efecto, los datos no evidencian un aumento en los ingresos del grupo beneficiario, pero sí muestran una caída significativa en los ingresos de las familias ajenas al programa, lo que sería atribuible a la crisis económica que afectó a las zonas donde se implementó la RPS. Todo esto indica que el programa está operando como un mitigador de las consecuencias adversas de una recesión. Al mismo tiempo, el porcentaje de gastos en alimentos de las familias del grupo de intervención se mantuvo en niveles altos ( $70 \%$ del gasto total), lo que implica que las transferencias no han tenido efectos sustantivos en los indicadores de pobreza.

También se observó que la participación en el programa promueve el consumo de una alimentación más diversa, tanto en la cantidad como en la calidad de los alimentos. Por ejemplo, las familias del grupo de intervención compran cuatro tipos más de alimentos que el grupo de comparación y tienden a gastar más recursos monetarios en alimentos de mayor calidad nutricional. Por otra parte, no hay evidencia de que el programa haya llevado a realizar inversiones para mejorar la vivienda o adquirir bienes durables, y tampoco se aprecia que la transferencia haya operado como un desincentivo al trabajo de los adultos.

En el ámbito de salud se observan efectos positivos del programa en el acceso a servicios de atención primaria. Aumentó el número de niños que pasó por controles del crecimiento y el de aquellos a los que se 
les administró sulfato ferroso. Sin embargo, tanto el grupo incluido en la RPS como el de comparación subieron de manera importante los niveles de vacunación, lo que sugiere que hubo un "efecto derrame" en la distribución de vacunas a las localidades y centros de salud del grupo de control.

Finalmente, la RPS tuvo un impacto significativo en la matrícula escolar. Estos efectos fueron más importantes en el grupo de 7 a 9 años y menos entre los niños de 12 a 13 años. Las diferencias de efectos fueron tres veces más relevantes para familias en situación de extrema pobreza y dos veces en el caso de las familias pobres, si se comparan con las familias no pobres. La diferencia en la retención escolar fue menor, aunque siguió siendo favorable a la RPS $(8 \%)$. Adicionalmente, se observó que la distancia hasta los centros escolares (costo de la escolarización) constituye un factor importante de la permanencia de los niños en la escuela, lo que subraya la necesidad de mejorar el acceso a la oferta.

\section{El Programa Oportunidades (ex ProgresA), de México}

El PROGRESA fue creado por el gobierno federal mexicano en 1997, como una estrategia para apoyar a familias rurales extremadamente pobres. En el 2001, durante la administración del Presidente Fox, pasó a llamarse Programa Oportunidades y amplió sus servicios a poblaciones urbanas. El Programa Oportunidades constituye uno de los casos más analizados y mejor evaluados en el ámbito de las transferencias monetarias condicionadas en América Latina, y su diseño marca cambios significativos en la provisión de servicios sociales en México. Esos cambios se expresan en los siguientes principios orientadores de la acción del programa: i) focalización, ii) intersectorialidad, iii) empoderamiento de las mujeres y iv) corresponsabilidad (Skoufias y Parker, 2001).

El propósito del Programa Oportunidades es incrementar las capacidades de familias en situación de extrema pobreza, a través de la inversión en capital humano. El programa tiene tres componentes: educación, salud y nutrición. En el componente educación, se entregan transferencias a familias con niños menores de 18 años de edad, matriculados entre el primer año de enseñanza primaria y el tercer año de educación secundaria. Con el objeto de generar incentivos para que las familias inviertan en capital humano, las transferencias se condicionan a la asistencia de los niños a la escuela. Si se registra una tasa mensual de inasistencia injustificada superior al $15 \%$, la familia no recibe la ayuda. El programa entrega recursos adicionales a las escuelas emplazadas en comunidades donde está en marcha, para compensar las externalidades negativas que podría generar el aumento de la demanda.

Los montos de las transferencias se determinan atendiendo a los ingresos adicionales que los niños habrían aportado a sus familias si hubieran estado trabajando. Los apoyos monetarios se incrementan a medida que aumenta la edad de los niños, y en la enseñanza secundaria, las transferencias son mayores para las niñas. Los montos se ajustan cada seis meses según la inflación, lo que evita el deterioro del valor real de la transferencia.

Por medio del componente salud, el Programa Oportunidades proporciona atención en salud primaria a todos los miembros de la familia. Estas prestaciones son entregadas por centros de la Secretaría de Salud y del Instituto Mexicano del Seguro Social. El componente nutrición contempla un apoyo monetario fijo para mejorar el consumo de alimentos, y la entrega de suplementos alimenticios destinados a niños de 4 meses a 2 años de edad, a lactantes y a mujeres que amamantan. Estos suplementos también se entregan a niños de 2 a 5 años que se encuentran en riesgo de desnutrición o mal nutridos. Para mantener los beneficios, las familias deben efectuar visitas periódicas a los centros de salud.

Los beneficios monetarios se entregan a la madre de familia. Se ha estimado que, en promedio, las transferencias monetarias entregadas representan el $22 \%$ de los ingresos mensuales de las familias participantes. Después de tres años de permanencia, las familias pueden solicitar la renovación de su condición de beneficiarias.

El procedimiento de focalización empleado por el Programa Oportunidades implica una selección geográfica a través de un índice de marginalidad construido con datos censales. Dentro de las comunidades seleccionadas, las familias elegibles se determinan mediante a un censo de todos los hogares de la comunidad. Finalmente, se selecciona a las familias beneficiarias en un proceso que involucra a la comunidad mediante la realización de asambleas.

$\mathrm{Al}$ respecto, se ha señalado que la aproximación del Programa Oportunidades a la selección de beneficiarios es eficiente en la identificación de áreas geográficas y familias extremadamente pobres, pero que es menos efectiva para diferenciar entre "localidades u hogares ubicados en la parte media de la escala". Por tal razón los programas de este tipo "pueden tener 
éxito en la reducción de la brecha de pobreza o en la severidad de la misma, pero es posible que su impacto en la proporción de hogares pobres sea mínimo" (Skoufias, Davis y De la Vega, 1999, p. 19).

Además, se ha planteado que "los costos no económicos asociados a la focalización ameritan considerarse a fondo para decidir si se debe emplear una estrategia de focalización a nivel de hogares. Las encuestas cualitativas de la evaluación de PROGRESA muestran que es probable que estos costos de focalización en las comunidades rurales, con frecuencia indígenas, no sean nada insignificantes" (Skoufias, Davis y De la Vega, 1999, p. 19). En ese sentido, González de la Rocha (2003) ha puesto de relieve la importancia de considerar qué efectos tienen los mecanismos de focalización utilizados en el capital social de las comunidades y familias beneficiarias.

González de la Rocha (2003) plantea que la selección errada de familias es una de las principales dificultades que enfrenta el Programa Oportunidades, por la insatisfacción que se genera entre las familias pobres que no fueron elegidas. Se podrían gestar "divisiones sociales al interior de las comunidades debido a que las percepciones sobre las condiciones propias y de las demás familias no corresponden con los identificadores técnicos del programa" (González de la Rocha, 2003, p. 12). Sin embargo, también se observan "relaciones de ayuda mutua e intercambio social, a pesar de la división entre familias beneficiarias y no beneficiarias" (González de la Rocha, 2003, p. 18).

Además, se han detectado dificultades en la selección de las familias mediante asambleas comunitarias, dado que en un número importante de comunidades estas asambleas no han funcionado en forma adecuada, porque los participantes no se atreven a manifestar sus opiniones abiertamente (González de la Rocha, 2003).

Uno de los aspectos distintivos del Programa Oportunidades ha sido su sistema de evaluación de impacto, que ha permitido contrastar hipótesis básicas para el diseño de los programas de transferencias condicionadas, como su costo-efectividad en comparación con otras modalidades de fomento del capital humano educacional (como el subsidio a la oferta), su incidencia en la distribución del tiempo de los niños (entre escuela, trabajo y tiempo libre) y sus efectos en la situación nutricional de los menores. Este sistema de evaluación considera métodos cuantitativos y cualitativos, que han permitido detectar los efectos del programa e identificar problemas y situaciones no previstas.
El diseño de la evaluación del Programa Oportunidades consideró la asignación aleatoria de 506 comunidades elegibles de siete estados a grupos de intervención y de control. Los 24.077 hogares existentes en las localidades de control e intervención fueron censados con anterioridad al inicio del programa, medición que constituyó la línea basal. Posteriormente, se comenzó a efectuar encuestas de seguimiento cada seis meses.

Skoufias y Parker (2001) encontraron que el programa ha tenido efectos positivos en el incremento de la matrícula escolar y en la disminución del trabajo de los niños, resultados que son mejores entre los adolescentes. Sin embargo, también observaron que los efectos en la reducción de la participación de los niños en actividades laborales fueron menores que los beneficios obtenidos en la escolarización. En el caso de las niñas, la mayor parte del éxito del Programa Oportunidades se dio respecto de adolescentes que combinaban la escuela con el trabajo doméstico, lo que podría indicar una disminución de su tiempo libre.

En el estudio cualitativo de González de la Rocha y Escobar (2002), se observó que una parte de las familias se manifestaba renuente a la permanencia de los niños en la escuela, actitud cruzada por distinciones de género. Estos autores indican que "había múltiples casos en los cuales los padres parecían buscar disminuir su propia carga doméstica con el trabajo de sus hijas" (González de la Rocha y Escobar, 2002, p. 27). También se detectó que un obstáculo importante eran las percepciones que tenían las familias pobres sobre el trabajo infantil. Algunos beneficiarios del Programa Oportunidades tendían a creer que el trabajo infantil: i) permite a los niños insertarse mejor en el mundo adulto; ii) evita la drogadicción, la participación en pandillas y el alcoholismo y, iii) supone un ahorro para la familia.

Al mismo tiempo, la participación en el programa ha generado algunos conflictos dentro de la familia. Ha habido separaciones y abandonos por parte de los hombres, así como bajas en el aporte de estos últimos a la economía familiar. Sin embargo, también hay solidaridad entre las mujeres para contrarrestar los efectos de las reacciones masculinas ante la pérdida de poder (González de la Rocha y Escobar, 2002).

Otro tema es el costo-efectividad de los subsidios condicionados a la demanda, en comparación con el incremento de la oferta (mejoramiento de la calidad de los servicios educacionales y/o construcción de más escuelas). Sobre la base del análisis de los datos del Programa Oportunidades, Coady y Parker (2002) 
concluyen que las intervenciones orientadas a la demanda tendrían un costo-efectividad de 9.730 pesos mexicanos por cada año extra de escolaridad generado, mientras que la expansión de la oferta alcanzaría un costo-efectividad de 113.500 pesos mexicanos por cada año adicional de escuela.

Por ultimo, Hoddinot y Skoufias (2003) analizaron los efectos en el consumo de calorías derivados de la participación en un subcomponente del Programa Oportunidades —las pláticas, que son actividades de

\section{IV}

\section{Conclusiones}

Los programas de transferencias condicionadas promueven la acumulación de capital humano entre los niños, niñas y jóvenes, con miras a quebrar el ciclo intergeneracional de la pobreza, y lo hacen creando incentivos para que las familias inviertan en capital humano educacional, nutricional y de salud. Estos programas surgen de un enfoque que privilegia la acción sobre las causas de la pobreza para lograr su superación en el largo plazo, a diferencia de los programas tradicionales de asistencia que se centran en la redistribución y la reducción de la pobreza en el corto plazo.

Dichos programas de transferencias atienden primordialmente a poblaciones en situación de pobreza estructural, entregando asistencia y procurando reducir riesgos futuros por la vía de la promoción del capital humano. En su mayoría, no fueron diseñados como redes de protección ante las conmociones económicas (Hicks y Wodon, 2001). Sin embargo, algunas iniciativas buscan explícitamente paliar las consecuencias de una crisis económica para los más pobres (Familias en Acción) y otras mitigaron los efectos de una recesión (la RPS de Nicaragua), lo que indica que las transferencias condicionadas pueden cumplir ese papel (Sedlacek, Hahi y Gustafsson-Wright, 2000; Rawlings, 2004). En todo caso, otros instrumentos, como los programas de empleos temporales, parecen ser más adecuados para enfrentar los peores efectos de las crisis (Morley y Coady, 2003)

La evidencia muestra que los programas de transferencias condicionadas son efectivos para incrementar la matrícula y la asistencia escolar en el corto plazo. Sin embargo, sus efectos en la reducción del trabajo infantil no son tan claros. El Programa Oportuni- educación nutricional-. Estos autores indican que, a pesar de que el grupo beneficiario de dicho programa incrementó la adquisición de alimentos ricos en calorías, este cambio es atribuible al aumento de ingresos generado por las transferencias. Sin embargo, las pláticas sí tienen efectos en la diversificación de la dieta y la obtención de calorías de productos animales, frutas y vegetales. Estos datos grafican la importancia que pueden tener las intervenciones psicosociales y educativas en los patrones de consumo de alimentos.

dades y el PETI logran disminuir de la participación de los niños en actividades laborales, pero en el caso del Programa Bolsa Escola la evidencia no es concluyente. Los elementos que marcan la diferencia podrían ser el componente específico del PETI que es la Jornada Ampliada, y el método utilizado por el Programa Oportunidades para establecer el monto de la transferencia.

Según Skoufias y Parker (2001), los datos del Programa Oportunidades muestran una relación inversa entre el trabajo infantil y la asistencia a la escuela, lo que indica que estas dos actividades son mutuamente sustitutivas. Sin embargo, la información sobre el Programa Bolsa Escola y y el Programa Oportunidades indica que es preciso hacer el seguimiento de la distribución del tiempo entre la escuela, el trabajo remunerado y no remunerado y el tiempo libre, para evitar que el logro de los objetivos escolares se obtenga a expensas del tiempo libre de los niños, o bien en coexistencia con el trabajo doméstico.

Un aspecto crucial para el diseño de los programas de transferencias condicionadas en educación es el de determinar el monto de la ayuda monetaria. Como se ha podido apreciar, los métodos difieren bastante de un programa a otro. Quizás la modalidad óptima para establecer un monto que permita fomentar la asistencia a la escuela y la eliminación del trabajo infantil, es estimarlo sobre la base del costo de oportunidad de enviar los niños a la escuela. Si se considera que este costo podría incrementarse con las edades de los niños y también podría resultar más alto para las niñas, lo razonable sería establecer transferencias mayores para los adolescentes y las niñas, tal como se hace en el Programa Oportunidades. 
Al definir el monto de la transferencia también debería considerarse el costo de la escolarización, es decir, todos los gastos asociados al envío del niño a la escuela. Este método es el empleado por Familias en Acción. Las transferencias educativas tendrían que ser entregadas en función del número de niños en la familia, para evitar la una posible pérdida de incentivos en los hogares más numerosos, y deberían ser reajustadas según la inflación, para evitar la caída de sus valores reales.

Un aspecto que debe tenerse en cuenta es el de las percepciones de los beneficiarios sobre el trabajo infantil y la educación. Los estudios efectuados en el PETI y el Programa Oportunidades encontraron que las familias atribuyen un valor limitado a la educación y no creen que el trabajo infantil sea perjudicial para el futuro de sus hijos. Esto muestra la importancia de efectuar intervenciones psicosociales encaminadas a cambiar estas representaciones. En caso contrario, al finalizar los programas, la probabilidad de que el niño retorne al trabajo y abandone la escuela podría ser alta, lo que amenazaría la sostenibilidad de los cambios.

La entrega prioritaria de las transferencias a las mujeres parece constituir una buena aproximación: la evidencia muestra que las mujeres administran las ayudas económicas de manera más eficiente que los hombres (IFPRI, 2002). Al mismo tiempo, esto les permite ganar sentimientos de autonomía e independencia, los cuales constituyen indicadores de empoderamiento (Zimmerman, 1990 y 1995). Sin embargo, es preciso evitar tanto las consecuencias adversas que podrían generarse por reacciones de los hombres ante la autonomía que adquieren las mujeres, como los conflictos derivados de una menor participación de las niñas en el trabajo doméstico.

Una buena práctica es la implementación de un componente de oferta que compense las mayores exigencias sobre el sistema escolar derivadas del aumento de la demanda. La información disponible indica que los subsidios a la demanda son bien recibidos por las escuelas, pero que los aprendizajes no mejoran a partir de la participación de los niños en los programas. Esto plantea la necesidad de aplicar medidas para mejorar la calidad de la educación que vayan más allá de una mera compensación ante el crecimiento de la demanda.

Hay indicios de que las transferencias condicionadas en apoyo de la demanda son más eficaces y eficientes que la expansión de la oferta para elevar los niveles educativos de los niños de familias pobres. Estos datos constituyen insumos para la toma de deci- siones, pero habrá que esperar los resultados de otras investigaciones. La información que proporcione el sistema de evaluación del Programa de Asignación Familiar en Honduras ayudará a tener un juicio más claro en la materia (Alvarez, 2001).

Los programas de transferencias condicionadas logran, en algunos casos, reducir la brecha de pobreza que afecta a las familias pobres, y en otros, mitigar las consecuencias de una crisis económica. Sin embargo, no está clara su potencia para lograr que los beneficiarios superen la línea de la pobreza, aunque se puede hacer presente que la probabilidad de que los sujetos se empinen por sobre esa línea dependerá del monto de la transferencia, de la focalización del programa y de la ausencia de contracciones económicas.

En el largo plazo, la superación de la pobreza dependerá del incremento de la capacidad de generar ingresos de las familias. Aunque las simulaciones indican ganancias salariales derivadas de más años de escolaridad equivalentes a un $8 \%$ para los niños del Programa Oportunidades y a un $9 \%$ para los niños de la RPS en Nicaragua (Morley y Coady, 2003: Skoufias y McClafferty, 2001), esta conversión no puede ser automática, debido a la incidencia de mediadores como la calidad del aprendizaje y las tasas de desempleo. En este sentido, resultan cruciales tanto el mejoramiento de la calidad de la educación como los vínculos con los programas orientados a la inserción laboral.

En cuanto a la salud y nutrición, el programa Familias en Acción tuvo efectos positivos en el acceso y uso de los servicios de la red de salud primaria. A su vez, la evidencia respecto del Programa Oportunidades indica que este tiene efectos positivos en la alimentación y también muestra que la incorporación de componentes psicoeducativos permite que las familias diversifiquen y mejoren la calidad de su dieta.

Por otra parte, los programas difieren en sus componentes, modalidades de administración y mecanismos de focalización, lo que fue más marcado en sus inicios. En los últimos años se ha tendido a la consolidación de programas con múltiples componentes (educación, salud y alimentación), administrados a nivel nacional y con mecanismos de focalización en varias etapas, siguiendo el modelo del Programa Oportunidades

Según la literatura sobre el tema, los programas de este tipo permiten una intervención más integral (debido a que atienden simultáneamente a las distintas dimensiones del capital humano), son más eficientes en sus procesos de planificación y gestión, y más eficaces en su focalización. Al mismo tiempo, sus 
intervenciones abarcan a la familia completa, por lo cual tienen el potencial para generar sinergias y ampliar el impacto.

La inclusión/exclusión de familias pobres dentro de las comunidades es un tema que deberá examinarse, atendiendo a los costos sociales y comunitarios que podrían derivar de tales prácticas. En este sentido, es importante considerar la experiencia del Programa Oportunidades en lo que se refiere a los sentimientos de insatisfacción apreciados en las familias que no fueron seleccionadas por el programa, situación que podría generar conflictos dentro de la comunidad y erosionar su capital social (González de la Rocha, 2003). Para atenuar los costos no económicos de la focalización habrá que perfeccionar los espacios comunitarios de participación, como las asambleas comunitarias del Programa Oportunidades o los consejos de control social del Programa Bolsa Escola.

Los organismos de la sociedad civil se han involucrado en alguna medida en la prestación de servicios, la planificación y la selección de los beneficiarios, tal como lo ilustra la experiencia de los consejos de control social del Programa Bolsa Escola. Una alternativa es la de subcontratar los servicios de organizaciones no gubernamentales experimentadas en el trabajo con poblaciones complejas, como los niños de la calle. Una condición para efectuar las transferencias podría ser la asistencia a programas ofrecidos por esas ONG, lo que permitiría maximizar la eficiencia de las intervenciones, al disminuir los costos asociados a la expansión de la oferta y utilizarse la experiencia de dichas instituciones.

Finalmente, lo que en definitiva marca una diferencia de estos programas de transferencias condicionadas con otras experiencias desarrolladas en América Latina, es la implementación de sistemas de evaluación de impacto como parte del diseño de los programas. Algunas de las evaluaciones han contemplado la asignación aleatoria de comunidades a grupos experimentales y de control, y mediciones repetidas. Los sistemas de evaluación han permitido establecer los efectos de las intervenciones y han facilitado la comparación de las hipótesis que se encuentran en la base de este tipo de programas. Particularmente rescatables son las experiencias del Programa Oportunidades (México), de la Red de Protección Social (Nicaragua) y del programa Familias en Acción (Colombia). También cabe mencionar el diseño del sistema de evaluación del Programa de Asignación Familiar (Honduras).
Alvarez, C. (2001): Evaluating a Safety Net Program. Family Allowance Program (PRAF) Honduras. Disponible en http:/ /www1.worldbank.org.sp.safetynets/

Attanasio, O., C. Meghir y otros (2004): Baseline Report on the Evaluation of Familias en Acción, IFS Reports, Londres, The Institute for Fiscal Studies, abril. Disponible en: http:// www.ifs.org.uk/

Attanasio, O., E. Battistin y otros (2005): How Effective Are Conditional Cash Transfers? Evidence from Colombia, IFS Briefing Notes, $\mathrm{N}^{\circ} 54$, Londres, The Institute for Fiscal Studies. Disponible en http://www.ifs.org.uk/

Banco Mundial (2001a): Assessment of the Bolsa Escola Programs, Sector Report, No 20208, Washington, D.C. Disponible en http//www1.worldbank.org/sp/safetynets/

(2001b): Eradicating Child Labor in Brazil, Sector Report, $\mathrm{N}^{\mathrm{o}}$ 21858, Washington, D.C. Disponible en: http://wwwwds.worldbank.org/servlet/WDS_IBank_Servlet?pcont= details\&eid=000094946_01122809560220

(2001c): Social Protection Sector Strategy: from Safety Net to Springboard, Washington, D.C., enero. Disponible en http://www1.worldbank.org.sp.safetynets/

Britto, T. (2005): Recent Trends in the Development Agenda of Latin America: An Analysis of Conditional Cash Transfers, Manchester, Reino Unido, Institute for Development Policy and Management (IDPM). Disponible en: http://www.eldis.org/ static/DOC17797.htm

CEPAL (Comisión Económica para América Latina y el Caribe) (2002): Panorama social de América Latina, 2001-2002,
LC/G.2183-P, Santiago de Chile. Publicación de las Naciones Unidas, $\mathrm{N}^{\mathrm{o}}$ de venta: S.02.II.G.65.

(2000): La brecha de la equidad: una segunda evaluación, LC/G.2096, Santiago de Chile.

Coady, D. y S. Parker (2002): A Cost-Effectiveness Analysis of Demand and Supply Side Education Interventions: the Case of Progresa in Mexico, FCND Discussion Paper, $\mathrm{N}^{\circ} 127$, Washington, D.C., Instituto Internacional de Investigaciones sobre Políticas Alimentarias (IFPRI). Disponible en http:// www.ifpri.org/

Departamento Nacional de Planeación (2003): Sinergia, Reportes de evaluación, $\mathrm{N}^{\mathrm{o}} 1$, Bogotá, D.C. Disponible en http:// www.dnp.gov.co/

Godoy, L. (2004): Programas de renta mínima vinculada a la educación: las becas escolares en Brasil, serie Políticas sociales, $\mathrm{N}^{\circ}$ 99, LC/L.2217-P, Santiago de Chile. Publicación de las Naciones Unidas, $\mathrm{N}^{\circ}$ de venta: S.04.II.G.137.

González de la Rocha, M. (2003): México: oportunidades y capital social, documento presentado al Seminario "Capital social y programas de superación de la pobreza: lineamientos para la acción" (CEPAL, Santiago de Chile, 10 y 11 de noviembre).

González de la Rocha, M. y A. Escobar (2002): Evaluación cualitativa del Programa de Desarrollo Humano Oportunidades, México, D.F, Centro de Investigaciones y Estudios Superiores de Antropología Social (CIESAS), diciembre. Disponible enhttp://www.progresa.gob.mx/

Hicks, N. y Q. Wodon (2001): Protección social para los pobres en América Latina, Revista de la CEPAL, No 73, LC/G.2130-P, Santiago de Chile, abril. 
Hoddinot, J. y E. Skoufias (2003): The impact of Progresa on food consumption, FCND Discussion Paper, No 150, Washington, D.C., Instituto Internacional de Investigaciones sobre Políticas Alimentarias (IFPRI). Disponible en http://www.ifpri.org/

Holzmann, R. y O. Jorgensen (2000): Manejo social del riesgo: un nuevo marco conceptual para la protección social y más allá, documento de trabajo, $N^{\circ}$ 0006, Washington, D.C., Banco Mundial. Disponible en http://www1.worldbank.org.sp. safetynets/

IFPRI (Instituto Internacional de Investigación de las Políticas Alimentarias) (2002): Nicaragua Social Protection Network. Pilot Phase Evaluation System: Impact Evaluation, Washington, D.C. Disponible enhttp://www.ifpri.org/

Morley, S. y D. Coady (2003): From Social Assistance to Social Development: Targeted Education Subsidies in Developing Countries, Washington, D.C., Instituto Internacional de Investigación de las Políticas Alimentarias (IFPRI).

Naciones Unidas (2001): Road Map towards the Implementation of the United Nations Millennium Declaration: Report of the Secretary-General, A/56/326, Nueva York. Disponible en http://www.worldvolunteersweb.org/

Ravallion, M. (2003): Targeted Transfers in Poor Countries: Revisiting the Trade-offs and Policy Options, Social Protection Discussion Paper, No 0314, Washington, D.C., Banco Mundial. Disponible en http://www1.worldbankorg/sp/safetynets/

Ravallion, M. y Q. Wodon (1999): Does Child Labor Displace Schooling? Evidence on Behavioral Responses to an Enrollment Subsidy, Policy Research Working Papers, $N^{\circ}$ 2116, Washington, D.C., Banco Mundial. Disponible en: http://econ.worldbank.org/docs/828.pdf

Rawlings, L. (2002): Colombia Social Safety Net Assessment, Washington, D.C., Banco Mundial Disponible en http:// www1.worlbank.org/sp/safetynets/

(2004): A New Approach to Social Assistance: Latin America's Experience with Conditional Cash Transfer Programs, Working Paper, No 30165, Washington, D.C.,
Banco Mundial. Disponible en: http://www1.worlbank.org/sp/ safetynets/

Secretaria do Programa Nacional de Bolsa Escola (2002): Manual do Programa Nacional de Bolsa Escola, Brasilia. Disponible en: http://www.mec.gov.br

Sedlacek, G., N. Hahi y E. Gustafsson-Wright (2000): Targeted Conditional Transfer Programs in Latin America: an Early Survey, Washington, D.C., Banco Mundial, junio. Disponible en http://www1.worlbank.org/sp/safetynets/

Sedlacek, G., Y. Yap y P. Orazem (2002): Limiting Child Labor Through Behavior-based Income Transfers: An Experimental Evaluation of the PETI Program in Rural Brazil, Washington, D.C., febrero. Disponible en http://www1.worlbank.org/ $\mathrm{sp} /$ safetynets/

Skoufias, E., B. Davis y S. de la Vega (1999): Suplemento al reporte definitivo: evaluación de la selección de hogares beneficiados por el Programa de Educación, Salud y Alimentación (PROGRESA) de México, Washington, D.C., Instituto Internacional de Investigación de las Políticas Alimentarias (IFPRI). Disponible en http://www.ifpri.org/:

Skoufias, E. y S. Parker (2001). Conditional Cash Transfers and Their Impacts on Child Work and Schooling: Evidence from the Progresa Programa in Mexico, FCND Discussion Paper, $\mathrm{N}^{\circ} 123$, Washington, D.C., Instituto Internacional de Investigación de las Políticas Alimentarias (IFPRI). Disponible en http://www.ifpri.org/

Skoufias, E. y B. McClafferty (2001): Is PROGRESA Working? Summary of the Results of an Evaluation by IFPRI, Washington, D.C., Instituto Internacional de Investigación de las Políticas Alimentarias (IFPRI).

Zimmerman, M. (1990): Taking aim on empowerment research: on the distinction between individual and psychological conceptions, American Journal of Community Psychology, vol. 18, $\mathrm{N}^{\mathrm{o}} 1$, Nueva York, Springer.

(1995): Psychological empowerment: issues and illustrations, American Journal of Community Psychology, vol. 23, $\mathrm{N}^{\circ} 5$, Nueva York, Springer. 